Horizons philosophiques

\title{
La forme du mouvement (sur la notion de rythme)
}

\section{Lucie Bourassa}

Volume 3, numéro 1, automne 1992

La fatigue culturelle du Canada français

URI : https://id.erudit.org/iderudit/800912ar

DOI : https://doi.org/10.7202/800912ar

Aller au sommaire du numéro

\section{Éditeur(s)}

Collège Édouard-Montpetit

\section{ISSN}

1181-9227 (imprimé)

1920-2954 (numérique)

Découvrir la revue

Citer cet article

Bourassa, L. (1992). La forme du mouvement (sur la notion de rythme). Horizons philosophiques, 3(1), 103-120. https://doi.org/10.7202/800912ar d'utilisation que vous pouvez consulter en ligne.

https://apropos.erudit.org/fr/usagers/politique-dutilisation/ 


\section{La forme du mouvement (sur la notion de rythme)}

Cette réflexion autour de la notion de rythme a été motivée par le désir d'expliciter le rôle qu'il joue dans l'organisation du sens des œuvres littéraires. Elle s'est imposée en particulier pour décrire des textes - poésies contemporaines, proses qui, ne présentant aucun signe évident de mesure régulière, semblent mettre en échec une conception du rythme fondée sur la régularité, qui a longtemps prévalu dans le domaine de la poétique.

\section{Une notion fuyante}

La notion de rythme traverse de nombreuses disciplines, de la physique à la linguistique, en passant par la biologie, l'anthropologie, la psychologie de la perception, l'esthétique, la musicologie et la poétique. La tentation est grande, alors, d'y voir un carrefour, un lieu de passage par lequel les divers savoirs pourraient communiquer, un concept-clé à l'aide duquel on pourrait mieux comprendre les rapports entre la physis d'un côté et l'homme de l'autre, celui-ci étant considéré à la fois dans ses modes d'appréhension du monde et dans ses productions symboliques.

Le problème est que le rythme (comme notion) semble très difficile à définir : on croit y avoir réussi, et voilà que les rythmes (empiriques) se mettent à excéder la définition. Paul Valéry, après avoir abordé la notion sous différents angles (comme loi de fonctionnement, système, mouvement, régularité, etc.), disait avoir "lu ou [...] forgé vingt définitions du rythme dont [il] n'adopt[ait] aucune ${ }^{1} "$. Plusieurs chercheurs, constatant les multiples divergences dans les théories, ont souligné le caractère fuyant de ce concept. C'est le cas du psychologue Paul Fraisse qui explique que les définitions souvent «partielles ou exclusives [...] ne permettent pas d'unir les acceptions différen-

1. CEuvres, Paris, Gallimard, "La Pléiade», p. 1289. 
tes» du rythme, en particulier parce que celui-ci n'est pas «un concept univoque, mais un terme générique ${ }^{2} »$. Cette plurivocité ne tient pas simplement au caractère générique de la notion : elle existe à l'intérieur même des disciplines. En poétique, par exemple, on en trouve des conceptions différentes, voire contradictoires : le rythme est tantôt vu comme régularité syllabique, accentuelle, etc. (comme mètre), comme distribution irrégulière d'un élément opposée à la disposition régulière d'un autre ou comme forme selon le déroulement temporel (sans référence obligée à la régularité).

Henri Meschonnic croit qu'il ne faut pas chercher à définir une notion de rythme applicable à tous les phénomènes naturels et à toutes les formes d'expression, parce qu'une telle généralisation entraînerait, selon lui, une réduction qui empêche de reconnaître la spécificité des manifestations empiriques du rythme, en particulier dans le discours. Dans les nombreuses définitions qu'il recense ${ }^{3}$, il repère, malgré les divergences, une dominante: celle du primat de l'identité à travers des variantes telles la périodicité, la répétition, l'isochronie, etc. Cette conception, héritée notamment de l'importance du mètre dans la tradition occidentale et de la confusion des champs d'application du concept de rythme, conduirait, en poétique, à faire entrer les textes dans un schéma abstrait. Meschonnic propose d'envisager le rythme poétique comme manifestation de l'oralité, de la temporalité et de l'historicité d'un sujetà travers l'organisation originale de marques à tous les niveaux du discours ${ }^{4}$, plutôt que comme schéma pré-existant aux œuvres. Cela semble permettre d'aborder immédiatement une question méthodologique concernant l'identification des éléments qui, dans un discours, auront un statut de marques, et plus globalement tous ceux qui constituent le rythme.

2. Les Structures rythmiques, Louvain, Publications universitaires de Louvain, 1956. Je dois cette référence à l'excellent chapitre de Jean-Jacques Nattiez sur «Rythme et mètre" en musique dans Musicologie générale et sémiologie, Paris, Christian Bourgois éditeur, collection "Musique/passé/présent", p. 311-338.

3. Voir Critique du rythme, Verdier, Lagrasse,1982, p. 149-176.

4. Ibid., p. 217. 
Mais le problème de reconnaissance des marques a bien vite fait de remettre à l'avant-plan celui de la définition : en effet, quelles propriétés nous permettront de qualifier une "organisation" de "rythmique" si l'on se refuse le recours à une mesure qui puisse la régler? Pour répondre, il m'a semblé nécessaire, malgré les inconvénients que cela présente, d'essayer de clarifier, de manière plutôt spéculative (en l'isolant provisoirement des constituants linguistiques), la notion de rythme, de dégager les propriétés qui le distinguent d'une autre forme d'organisation. Cette réflexion aura cependant comme horizon la possibilité d'expliciter des fonctionnements du discours et ne tentera donc pas de résoudre la plurivocité du concept en vue d'en «unir les diverses acceptions». Elle soulèvera par contre des difficultés et des contradictions qui sous-tendent les définitions et débordera ainsi à l'occasion le champ de la poétique.

Le célèbre article d'Émile Benveniste, "Le rythme dans son acception linguistique ${ }^{5}$ ", consacré àl'étymologie de "rhuthmos" et à l'historique de son sens dans le monde grec («chez les auteurs ioniens et dans la poésie lyrique et tragique, puis dans la prose attique, surtout chez les philosophes ${ }^{6}$ ", fournit un point de départ intéressant pour une telle discussion. Même si le sens de rhuthmos s'est beaucoup modifié des loniens à Platon, les

5. Dans Problèmes de linguistique générale, t. I, Paris, Gallimard, 1966, p. 327-335. Cet article est devenu presque une référence obligée des gens qui s'intéressent aux problèmes de rythme poétique. Outre Henri Meschonnic, qui le commente abondamment (dans Critique...), signalons aussi qui le mentionnent ou le discutent : M. Blanchot, Le Livre à venir, Paris, Gallimard, 1969; L'Écriture du désastre, Paris, Gallimard, 1980; M. Deguy, "Figure du rythme, rythme des figures", Langue française, $n^{\circ} 23$, "Poétique du vers français", éd. H. Meschonnic, (1973-1974), p. 24-40; Choses de la poésie et affaire culturelle, Paris, Hachette, 1986; L. Jenny, La Terreur et les signes; poétiques de rupture, Paris, Gallimard, "Les Essais" (CCXXII), 1982; J. Garelli, "Discontinuité poétique et énergétique de l'être", La Liberté de l'esprit, n 14, "Qu'est-ce que la phénoménologie?", Paris, Hachette (hiver 1986-1987), p. 25-53; H. Morier, Dictionnaire de poétique et de rhétorique, $4^{\circ}$ édition revue et augmentée, Paris, PUF, 1989 [première édition 1961]; M. Collot, "Rythme et mètre; entre identité et différence", Protée, vol. 18, n 1, "Rythmes", éd. L. Bourassa (hiver 1990), p. 75-80.

6. Ibid., p. 328. 
définitions rapportées par Benveniste concernent toutes l'un ou l'autre des problèmes du rythme qui sont au coeur des débats actuels. L'historique du linguiste servira à les mettre en lumière et non à fournir un argument étymologique à même de prouver la vérité de telle ou telle définition à partir de l'origine du mot.

\section{Les conceptions naturelle et anthropologique du rythme}

La première discussion de Benveniste vise à rectifier l'étymologie traditionnelle de rhuthmos, qu'on dérivait de rhéô ("couler»), pourl'interpréter comme «mouvement plus ou moins régulier des flots ${ }^{7}$ ». Benveniste affirme que nous «métaphorisons aujourd'hui quand nous parlons du rythme des flots ${ }^{8}$ ” et que c'est une erreur de montrer l'origine d'un sens actuel du mot rythme, "considération de "temps", d'intervalles et de retours pareils $s^{9}$ par un lien sémantique entre rhéô et le mouvement de la mer. Exposant les significations du terme dans l'Antiquité, le linguiste démontre que la reconnaissance du «principe cadentiel» est le fruit "d'une lente élaboration ${ }^{10}$ » et non d'un apprentissage spontané du "principe des choses" à partir de la nature. Ici, Benveniste pose, au-delà du problème philologique, une question philosophique "concernant le rapport entre une chose et la représentation de son sens", comme le fait remarquer Michel Deguy ${ }^{11}$. II fait ainsi ressortir l'existence de deux conceptions opposées du rythme. La première l'envisage comme un phénomène naturel sur lequel s'appuieraient les activités humaines : de nombreux théoriciens, poéticiens et musiciens fondent la nécessité des rythmes dans l'art, si complexes soient-ils, dans un rapport avec une battue originelle, qu'elle soit cosmique ou

7. Ibid., p. 327-328.

8. Ibid., p. 335.

9. Ibid., p. 327.

10. Ibid., p. 335.

11. Choses..., p. 104. 
physiologique; la seconde (qu'adopte ici Benveniste) considère que la notion de rythme servirait à "caractériser distinctivement les comportements humains ${ }^{12}$ " et que la reconnaissance de rythmes naturels serait le fruit d'une projection de la "conscience des durées et des successions qui les règlent ${ }^{13}$ ".

Cette distinction est d'une importance capitale car l'une ou l'autre vision implique une attitude différente dans la poétique du rythme. Meschonnic a montré certains écueils de la première : expliquer tous les rythmes par un principe naturel peut conduire à un amalgame du culturel, de l'historique et du naturel, à la confusion, par exemple, de pratiques ponctuellement codées avec une contrainte physiologique ou cosmique. Confusion que l'on a faite, entre autres, au sujet de l'alexandrin, expliquant que sa longueur était idéale parce qu'accordée "naturellement» auxpossibilités respiratoires. Ce qui peut avoir pour conséquence l'effacement de l'historicité de certains choix esthétiques, mais aussi celui des conduites subjectives au profit d'une identité totalisante et généralisante.

II est certes contestable, historiquement, non pas de faire dériver rhuthmos de rhéôt ${ }^{14}$, mais d'associer, par là, le rhuthmos au «mouvement des flots", d'affirmer que tel ou tel phénomène naturel a engendré l'idée de rythme, et par la suite de comprendre toute manifestation rythmique comme reproduction d'un rythme naturel. Toutefois, il y a lieu de s'interroger sur les raisons de la permanence de tels «faits principiels ${ }^{15}$ » dans les théories du rythme. Meschonnic y voit la persistance d'une "théorie traditionnelle» qui pense en termes d'origine plutôt que de fonctionnement, de nature plutôt que d'histoire. Cela est vrai si l'on considère les phénomènes naturels (mouvements des

12. Problèmes..., p. 327.

13. Ibid.

14. "Le rapport de rhuthmos à rhéó ne prête par lui-même à aucune objection", explique Benveniste à la p. 332 qui ajoute que c'est l'interprétation de rhuthmos comme "mouvement des flots" qui est erronée, car, dans l'antiquité, ce mot n'a jamais été employé dans ce sens.

15. Comme le fait remarquer Deguy dans Choses ..., p. 105. 
flots, battements du coeur, scansion de la marche, etc.) comme l'origine et la condition de rythmes artistiques qui ne feraient que les répéter. Mais il y aurait peut-être lieu de comprendre autrement le rapport de ces "faits principiels" aux rythmes des pratiques symboliques, ainsi que le suggère Deguy lorsqu'il émetl'hypothèse que les phénomènes naturels sont des grands "comparants" grâce auxquels la pensée peut se «figurer", avoir une «image» de ce qu'elle conçoit dans le langage :

Dans tous les cas le fait principiel est une image. Et il ne devrait pas tants'agir de dériver réellement et inductivement les rythmes (musicaux, gestuels, poétiques) que de comprendre grâce à quel schème originairement symbolique, c'est-à-dire valant métonymiquement pour le tout, la pratique et la pensée du rythme se (res)saisit pour elle-même ${ }^{16}$.

Les comparants «naturels» ne font peut-être qu'illustrer une propriété fondamentale du rythme, la création de différences repérables, la distinction qui conditionne la perception. Le "cadentiel" du coeur, du souffle, de la marche ou de la mer donnent un «fruste modèle empirique du rythme ${ }^{17}$ ». Ils fournissent l'image de "cellules diacritiques minimales": coup, silence; levé, posé; avancée, recul, auxquelles s'ajoutent divers paramètres : intensité (coups faibles ou forts), durée (longueur, brièveté, rapidité, lenteur), proximité ou éloignement, etc. Dans cette «imagination générale du rythme», Deguy repère un schéma qui "articule deux marques différentes d'un "accent" irréductible à la quantité, et deux silences différenciés comme plus ou moins longs ${ }^{18}$ ". Propriétés simples du rythme, ces relations entre les marques et les pauses se multiplieront dans les productions humaines par une grande variété de positions des éléments.

\section{Le rythme : loi ou phénomène?}

Après avoir réfuté l'interprétation étymologique tradition-

16. Ibid.

17. M. Deguy, "Figure ...", p. 34.

18. Ibid. 
nelle du "rythme comme la mer», Benveniste expose les significations de rhuthmos avant que la notion ne prenne, chez Platon, le sens de "considération d'intervalles et de retours pareils". Le premier sens qu'il retient est celui de «forme», lui donnant les attributs de distinctivité, de différenciation ainsi que d'organisation, $d$ ' "arrangement caractéristique des parties dans un tout ${ }^{19}{ }^{\prime}$. Le terme recouvre des emplois fort variés, de la forme des "signes de l'écriture ${ }^{20}$ " à celle d'une chaussure ${ }^{21}$ et à celle des «institutions ${ }^{22}$ "; de la différence entre des éléments ${ }^{23}$ à la " "forme" individuelle et distinctive du caractère humain ${ }^{24}$ ". II s'y greffe aussi, à travers les dérivations, le sens de proportion: "arrhuthmos pour une passion "disproportionnée"25". Le rhuthmos, "forme distinctive; figure proportionnée; disposition $^{26}$ " était dans l'Antiquité une notion très vaste, tout comme l'est aujourd'hui le rythme.

La traduction de rhuthmos par «forme» ne permet pourtant pas de distinguer cette notion de celles appelées par d'autres termes comme «skhèma, morphè, eidos ${ }^{27}$ ". Benveniste revient alors à l'étymologie pour préciser sa définition. II décompose le terme en rhéin et -thmos, et les deux développements qui s'ensuivent confèrent à la notion toute son originalité. "La formation en -thmos, écrit-il, mérite attention pour le sens spécial qu'elle confère aux mots "abstraits". Elle indique, non l'accomplissement de la notion, mais la modalité particulière de son accomplissement, telle qu'elle se présente aux yeux ${ }^{28}$ ". Cela indique la spécificité d'un acte ou d'un accomplissement circonstancié, par exemple «la danse particulière vue dans son

19. Problèmes..., p. 330.

20. Ibid.

21. Ibid.

22. Ibid., p. 329.

23. Ibid., p. 328.

24. Ibid., p. 330 .

25. Ibid., p. 331.

26. Ibid., p. 332.

27. Ibid.

28. Ibid. 
développement ${ }^{29}$ 》 et implique une perception, quelque chose qui "se présente aux yeux» ou aux autres sens. Rhéin permet de différencier rhuthmos de skhèma en opposant la mobilité du rythme à la fixité de la forme. Rhuthmos désigne donc :

La forme dans l'instant qu'elle est assumée par ce qui est mouvant, mobile, fluide, la forme de ce qui n'a pas de consistance organique : il convient au pattern d'un élément fluide, à une lettre arbitrairement modelée, à un péplos qu'on arrange à son gré, à la disposition particulière du caractère ou de l'humeur ${ }^{30}$.

La valeur de "distinctivité" présente dans la première définition s'enrichit par celle de «mouvement». Le rythme est non seulement l'arrangement qui distingue, mais l'arrangement distinctif de ce qui se meut.

Benveniste souligne le lien de cette notion avec la philosophie ionienne, dans laquelle elle avait une valeur ontologique, puisque le rhéin y était vu comme "le prédicat essentiel de la nature des choses". II est curieux que le linguiste constate, d'une part, que rhuthmos servaità "caractériser distinctivement les comportements humains" et, d'autre part, qu'il avait une "valeur ontologique" par son lien étymologique avec «le prédicat essentiel de la nature des choses". La nouvelle définition du rythme comme "manière particulière de fluer" me semble privilégier, plutôt que les "comportements humains" par opposition aux "phénomènes naturels», l'indissociabilité du rythme avec l'apparaître par opposition à quelque antériorité du rythme face à toute manifestation. Toutefois, les deux oppositions se recoupent souvent dans des théories, car les phénomènes naturels sont habituellement envisagés non comme apparaître et surgissement de formes mais comme une grande loi universelle. Laurent Jenny dit que beaucoup de théories considèrent soit le rythme, soit le mètre comme "un mécanisme transcendant qui se laisse penser avant toute expression, toute mise en 
œuvre du discours ${ }^{31}$ » en donnant les exemples de Claudel et de Jousse. Chez le premier, il voit une métaphysique du mètre ${ }^{32}$ :

Chez Claudel derrière [des] concessions à la dimension vivante du rythme, il y a un double primat du mètre. Primat par antériorité : le mètre est l'ordre éternel. Primat par supériorité fonctionnelle : le mètre est moteur et contrôle du rythme [...]. Le coeur donne donc l'siambe fondamental», le diapason métrique, à la fois mesure-origine et mesure-étalon. Au souffle ensuite de broder sur ce patron métrique, d'y façonner la part intelligente du rythme [...]. Le rythme est là pour [...] ranimer toute la tension. Il est si bien asservi à cette fonction qu'il se définit à peine différemment du mètre : "ll consiste en un élan mesuré de l'âme répondant à un nombre toujours le même qui nous obsède et nous entraîne." La part humaine de l'activité rythmique est elle-même marquée d'un chiffre divin. [...] Ainsi, dans la théorie de Claudel, les poumons se donnent l'illusion de la vie, mais, depuis toujours, c'est le métronome divin qui tient le soufflet ${ }^{33}$.

II voit chez le second une "métaphysique du rythme»: "Jousse propose de rapporter le rythme à "une loi de l'oscillation universelle", dont les racines les plus profondes plongeraient dans le métabolisme dynamique de la vie cellulaire ${ }^{34}$." Dans ces deux théories, "le rythme ne se produit pas : il n'est que l'écho d'un grand principe toujours déjà là ${ }^{35}$ ".

La "mémoire étymologique" de rhuthmos, telle que Benveniste l'a "réactivée", invite à penser le rythme comme "production de la forme $e^{36}$ ". Cette indissociabilité du rhuthmos et du surgissement d'une forme permet de concevoir la mise en rythme et la mise en sens comme un même mouvement,

31. La Terreur..., p. 223.

32. II faudrait toutefois ici distinguer la théorie et la pratique poétiques de Claudel, ainsi que diverses époques de la pratique.

33. Ibid., p. 224.

34. Ibid.

35. Ibid., p. 225.

36. Ibid., p. 226. 
ouvrant ainsi tout un champ de possibilités à la poétique. Une pensée du rythme comme phénomène concomitant de l'apparaître pourrait aussi devenir féconde dans une réflexion sur d'autres arts.

\section{La modalité du mouvement : une dynamique temporelle?}

Les phénomènes naturels, comme le suggère Deguy, peuvent offrir une sorte d' «image primitive» du rythme; en tant que "comparants", ils illustrent quelques propriétés diacritiques de l'apparaître en mouvement. Mais les rythmes de tel poème, ou même de telle sonate, ne se modèlent pas nécessairement sur un phénomène naturel ou une loi qui leur serait antérieure. Car le rythme "tel qu'en lui-même" les œuvres le réaliseraient ou le changeraient n'a pas d'existence autonome. Le compositeur André Souris dit que «la notion de rythme en soi est une notion vide" et qu'«il n'y a de rythme que de quelque chose, et particulièrement de quelque chose qui sonne ${ }^{37}$ ". Quelque chose qui sonne, pour la musique, ou plus généralement, quelque chose qui apparaît. S'il y a des rythmes dans les phénomènes naturels, c'est que ces rythmes sont «la modalité particulière» de leur "accomplissement» "telle qu'elle se présente aux yeux", à l'ouïe, etc.

Souris oppose aux définitions du rythme comme «loi de régularité, de symétrie, d'isochronisme ${ }^{38}$ " subordonnant toute production, celle d'un "phénomène à la fois complexe et cohérent dont la complexité et la cohérence singulières engendrent sa durée propre, spécifique, essentiellement qualitative ${ }^{39}$ ". Le rythme émane pour lui d'un mouvement musical particulier, d'une organisation de l'œuvre et de ses constituants sonores, dont se dégage une temporalité sensible. Les rapports de durée qui le constituent sont qualitatifs, ils ne se séparent pas de la matière sonore elle-même : hauteur, intensité et timbre. Souris

37. André Souris, "Rythme», François Michel éd., Dictionnaire de la musique, t. III, Paris, Fasquelle, 1961, p. 606.

38. Ibid., p. 605.

39. Ibid., p. 606. 
établit une relation entre trois termes : "structuration», "durée", "sons", qui sont à la fois "agents" et "produits" des deux autres :

Soit une structure mélodique, de tempo lent, d'abord percutée sur un xylophone et soutenue par une clarinette. De part et d'autre, un même schéma de durées organise le déroulement des intervalles et le temps chronométrique de la mélodie. On ne peut s'en tenir à constater que la même mélodie et le même schéma rythmique ont été exécutés en staccato, puis en legato au niveau de la perception sensible, non seulement le jeu de l'instrument et leurs qualités propres affectent la mélodie de caractères hétérogènes, mais le temps de son déroulement acquiert dans chaque exécution une dimension spécifique [...]. L'élasticité de ce temps s'éprouve en modifiant les différents facteurs qui l'engendrent ${ }^{40}$.

II y va donc du caractère propre, singulier, sensible du rythme-temps qui est coextensif à une œuvre (ici musicale); ce caractère se trouve réduit lorsqu'on décrit le mode de mouvement uniquement à partir de durées quantitatives ou de quelque loi antérieure.

Concevoir le rythme comme «rythme de quelque chose» et non comme une loi antérieure invite à prendre en compte, lorsqu'on l'étudie dans des œuvres, «tous les aspects de ses manifestations structurantes". Le rythme discursif ne se confond pas avec le rythme musical : dans l'apparaître du texte, il y a le sens, ce qui n'est pas le cas de la musique; dans la "disposition" des sons, il y a souvent un "compte» de valeurs proportionnelles des durées, ce qui n'est pas dans le langage (du moins, pas dans les langues où la durée n'est pas phonologique : excepté pour les comptines ou la poésie chantée qui se règlent alors sur une battue musicale). Dans un texte littéraire, il ne saurait être question, comme en musique, d'étudier le rythme selon les «points de vue des échelles, des modes, de la tonalité, de la non-tonalité, de la monodie, des formes

40. Émile Benveniste, Problèmes..., p. 606. 
contrapuntiques, de l'harmonie, des instruments, des intensités, des densités polyphoniques, des registres de l'accentuation, du phrasé ${ }^{41}$ ». Mais la compréhension du rythme comme dynamique relationnelle, exposée par Souris, est intéressante : l'interaction de constituants sensibles produit une temporalité singulière, qui elle-même dispose nouvellement la qualité sonore (qui sera la "signifiance" et le fonctionnement de la signification en poésie).

Mais en rattachant le rythme et la temporalité, n'avons-nous pas débordé de la notion la plus ancienne, décrite par Benveniste, pour basculer dans la notion "platonicienne»? Le linguiste insiste d'abord sur le caractère temporaire et modifiable de la forme telle que désignée par rhuthmos, forme "sans fixité ni nécessité naturelle et résultant d'un arrangement toujours sujet à changer ${ }^{42}$ » qui la démarque du rythme platonicien dans lequel est introduite l'idée d'une régulation du mouvement. À la fin de son article, il insiste sur la spatialité de la notion "originale" et prête au terme platonicien seulement une dimension temporelle. Mais peut-on concevoir l'écoulement, le mouvement, la transformation dans le "flux", sans faire appel à l'intuition du temps? Michel Deguy se demande, avec pertinence, "comment auraient été chronologiquement, réellement, espacées une représentation de la spatialité et une de la durée, s'il s'agit des formes de l'intuition sensible de quoi que ce soit, pour parler avec Kant ${ }^{43}$ "? Jacques Garelli s'inspire de l'acception ionienne pour établir un rapport entre rythme et temporalité :

\footnotetext{
[...] si [...] la formation en - (q)moV indique non l'accomplissement de la notion, mais la modalité particulière de son accomplissement, telle qu'elle se présente "aux yeux», il n'est pas surprenant, dès lors, que la problématique du temps s'inscrive au coeur de celle du rythme, de par la "configuration" et la uforme" du déploiement de cet accomplissement, pour autant qu'on soit conduit à admettre qu'il
}

41. André Souris, "Rythmes...", p. 607.

42. Problèmes..., p. 333.

43. Choses..., p. 108. 
n'y a pas de déploiement sans un acte temporalisateur qui l'effectue ${ }^{44}$.

Meschonnic conçoit le rythme comme «temporalité subjective» et "forme-sens du temps ${ }^{45}$ ». Mais la temporalité rythmique, telle que la décrivent (différemment) Garelli et Meschonnic (en poésie) et Souris (en musique), n'est pas celle des durées de l'horloge. Elle concerne une dynamique de relations entre des éléments qui donnent à une œuvre sa configuration.

\section{Les propriétés d'une manière particulière de fluer}

Quelles seraient les propriétés qui permettent la saisie d'une «manière particulière de fluer», "telle qu'elle se présente aux yeux", à l'ouïe, etc.? Que désigne la forme "sans fixité ni nécessité naturelle et résultant d'un arrangement toujours sujet à changer» par laquelle Benveniste définit le rhuthmos? Comment peut-on penser cette modification, ce mouvement? Est-ce simplement l'organisation d'un ensemble tel qu'il se présente dans un instant, mais ne sera pas le même l'instant suivant, ce qui le différencierait de la skhèma et de la morphè qui seraient, elles, dotées d'une propriété de permanence? On ne cherchera pas, ici, de réponse historiquement satisfaisante à cette question puisque ce n'est pas là notre propos. Mais le conceptionien, dans sa formulation benvenistienne, suggère plusieurs développements qui pourraient s'avérer utiles pour une poétique du rythme. Les "configurations", "résultant d'un arrangement toujours sujet à changer", évoquent la forme (spatiale) dans l'instant ainsi que les formes successives. Mais la «manière particulière de fluer" souligne davantage la modalité du processus de transformation lui-même, ajoute à l'idée de modification constante celle d'un principe distinctif de modification ou, du moins, celle de caractéristiques spécifiques à un mouvement qui permettent de le distinguer d'un autre. Benveniste parle aussi du "pattern d'un élément fluide», pattern qui peut se

44. "Discontinuité....", p. 27-28.

45. Critique..., p. 224. 
concevoir dans la spatialité mais aussi dans le déroulement, la temporalité. Or, si on accepte d'intégrer cette idée d'un pattern, même si c'est un pattern qui change, on doit s'interroger sur ce qui permet la reconnaissance d'une telle structuration dans un flux.

Après avoir défini le rhuthmos présocratique, Benveniste évoque celui de Platon avec sa loi des nombres. II rappelle ensuite, sans le commenter, un autre texte, mystérieux, de Platon, où il est dit que de la même manière que «l'harmonie est une consonance, la consonance un accord [...] le rythme résulte du rapide et du lent, d'abord opposés, puis accordés ${ }^{46}$ ". L'introduction du paramètre du tempo convient à une exécution musicale, chorégraphique ou théâtrale, mais devient arbitraire pour un texte écrit. Toutefois, la formulation de Platon permet de poser deux problèmes intéressants. D'abord, par analogie avec la "proximité" ou «l'éloignement" des sons dans le rapide et le lent, elle invite à penser la proximité et l'éloignement des marques rythmiques, le resserrement ou l'extension des intervalles, le jeu de différences généralisées entre «conjonctions" et «disjonctions", dont Michel Deguy esquisse une théorisation à propos de la synérèse et de la diérèse, qui serait à développer sur des segments de discours plus vastes ${ }^{47}$. Ensuite, on observe dans cette dernière proposition quelque chose d'étrange à faire résulter le rythme d'une opposition, puis d'un accord, entre le rapide et le lent ${ }^{48}$. Cela pourrait s'appliquer d'abord à une alternance puis à une simultanéité. Différence et homologie viennent se comparer à la consonance et à l'harmonie : cela pourrait donc signifier l'idée d'un ordre, de proportions quasi mathématiques entre le rapide et le lent - analogues à celles qui régissent la formation des accords sur le plan des «hau-

46. Voir dans Le Banquet, 187 b; Benveniste cite ce passage à la p. 334.

47. Voir notamment dans "Figure ...", et dans la version remaniée de ce texte «Figurer le rythme" qui apparaît dans Choses...

48. Michel Deguy remarque que le "passage" d'une "différence" à une "homologie» est en lui-même difficile à élucider, voir Choses ..., p. 108. 
teurs". Ce qui peut davantage que l'ordre mathématique, devenir intéressant pour une poétique du rythme, c'est la possibilité de comparer ce qui se succède et de percevoir une relation qui s'établit à partir d'une différence et d'une parenté entre les segments, les morceaux d'un déroulement. Ceci se rapproche d'une hypothèse de Valéry: "Dans le rythme, le successif a quelques propriétés du simultané ${ }^{49}$." La clé de la saisie d'une "manière particulière de fluer", d'un principe d'organisation des éléments dans le déploiement, ne reposerait-elle pas sur la création de relations entre éléments successifs qui leur donnent "quelques propriétés du simultané»? Le rythme ferait alors appel à la mémoire et nous conduirait ainsi à repenser la distentia augustinienne. Augustin se demande, après avoir récité un vers, comment s'opère la perception de la différence entre les brèves et les longues :

\begin{abstract}
Qu'est-ce que je mesure? Où est la brève qui est ma mesure? Où est la longue que je mesure? Toutes les deux ont retenti, elles se sont envolées, elles ont passé, elles ne sont plus : et voilà que je les mesure et réponds avec assurance, autant qu'on peut se fier à un sens exercé, qu'évidemment l'une est simple, l'autre double en durée. Mais je ne le puis que si elles sont passées et achevées. Ce n'est donc pas elles que je mesure, mais quelque chose qui demeure gravé dans ma mémoire ${ }^{50}$.
\end{abstract}

C'est la présence du texte, son altérité, qui permet à Augustin de prendre conscience du «temps» et c'est sa mémoire qui lui rend possible l'attribution de valeurs proportionnelles aux durées syllabiques. Le rapport syllabique mesurable du simple au double n'est pas pertinent en langue française, non plus que dans plusieurs autres langues modernes; ce n'est pas lui qui me retient ici, mais la possibilité de reconnaître une "manière spécifique de fluer», qui pourra s'appuyer sur quelques éléments minimaux de retours. Ce minimum d'itération -

49. Cahiers, t. I, Paris, Gallimard, "La Pléiade", p. 173.

50. Saint Augustin, Les Confessions, livre XI, Paris, Garnier, coll. «GF», p. 278. 
qui devrait permettre la perception de relations entre ce qui précède et ce qui suit dans un mouvement, la perception d'une modalité propre aux différenciations successives - impose-til une mesure régulière? Beaucoup de théories répondent oui à cette question et définissent le rythme comme dialectique du même et de l'autre en donnant à même le sens de mètre. Mais une telle réponse conduit à exclure les discours non métriques du rythme ou, alors, à élargir considérablement le sens du mot "mètre». Qui n'a senti, intuitivement, une différence entre la manière de fluer des proses de Proust et de Céline, ou encore de Marguerite Duras? Entre le Coup de dés et tel poème de Sources du vent? La double réflexion - sur le jeu des conjonctions et disjonctions d'une part et sur l'importance de la mémoire dans la saisie des transformations du mouvement d'autre part - provoquée par la proposition énigmatique de Platon, permet plutôt de formuler ce qui suit, à propos du rythme poétique : des éléments contrastants d'un côté, et des éléments récurrents de l'autre, créeront (compte tenu des contraintes phonologiques et syntaxiques de la langue de l'œuvre) dans la structuration d'un texte, une rythmique signifiante selon leurs positions : rapprochement ou éloignement, conjonction et disjonction, mais aussi "positions» par rapport aux autres éléments du discours. Une manière particulière de fluer implique un double processus de différenciation (qui qualifie le flux et suscite divers types de groupements) et de comparaison (entre points qualifiés et groupes). Chacun de ces processus, qui ne sont pas séparés en pratique, fait appel aux contrastes et aux retours.

Des rapports s'établissent entre unités de sens marquées (accents, fins de vers ou de segments graphiques, retours phonétiques, lexicaux, syllabiques-accentuels, etc.) surtout entre les rappels, ce qui fait associer les unités de sens au-delà de leur succession immédiate. On peut donner un bref exemple à l'aide d'une phrase de Jacques Borel :

\footnotetext{
${ }^{1}$ Comme si, ${ }^{2}$ par ces haltes devant des stèles dont elle sait bien dans le même temps qu'elle n'a que faire, ${ }^{3}$ la mémoire, ${ }^{4} \mathrm{~d}$ 'instinct pour tout pèlerinage prête à appareiller, ${ }^{5}$ se sentait en ses propres assises plus profondément
} 
enracinée, ${ }^{6} \mathrm{si},{ }^{7}$ de ces signes rituels qui sont ceux aussi de toute fête, ${ }^{8}$ elle tenait la promesse ou l'espoir d'une illumination elle-même comme redoublée ${ }^{51}$.

L'auteur parle ici de l'attachement qu'il porte à ces signes, peut-être puérils mais non dénués de sens, que sont les dates qui apparaissent sur les lettres, ou dont il marque chacun des livres achetés, lus. Dans cette phrase, des figures phonématiques rapprochent la mémoire de ces halteset stèles (les dates), dont elle sait bien dans le même temps qu'elle n'a que faire...; d'autres motivent la nécessité, pour la mémoire (pour tout pèlerinage, pour appareiller), de se fonder sur ses propres assises, d'être prête, plus profondément enracinée, si elle veut s'élancer, "tenir» la promesse et l'espoir. Une série d'échos en /s/ et en /i/ renvoient au Comme si initial "redoublé" par le si médian, posant une possible condition de l'illumination dans les assises et l'enracinement de la mémoire, grâce à ces signes rituels que sont les stèles.

Dans la linéarité du discours, des relations, de ressemblance ou de contraste, s'établissent entre différents types de groupes sur la base de leur longueur, de leur qualité suspensive ou conclusive, etc. La phrase de Borel se divise, notamment, en sept groupes suspensifs et un conclusif ${ }^{52}$. Les sept premiers segments se ressemblent, par la pause et la suspension de voix qu'ils exigent, mais contrastent par leur longueur : trois groupes brefs se détachent. Comme siet simettent en relief le caractère hypothétique de l'effet des traces (des dates) sur la mémoire; un autre comme, juste avant la chute, vient «redoubler» le premier. Le groupe la mémoire, sujet attendu de cette ample phrase, apparaît d'autant plus marqué qu'il suit un très long segment.

51. Commémorations, Paris, Le Temps qu'il fait, 1990, p. 34. Les chiffres numérotent les séquences ponctuées pour les besoins d'une explication qui suivra.

52. Les groupes suspensifs sont ceux qui demanderaient, dans une lecture à haute voix, que l'intonation, en leur fin, s'élève légèrement, ou en tout cas ne baisse pas; les groupes conclusifs sont au contraire ceux qui imposent une chute de voix. On pourrait analyser un autre niveau de segmentation, inférieur, celui des mots phonologiques, c'est-à-dire des unités syntaxiques porteuses d'un accent tonique final. 


\section{Conclusion}

L'article de Benveniste a permis de relever plusieurs problèmes liés à la notion de rythme. Des oppositions ont été évoquées qui sont souvent à la source des querelles et polémiques entre les diverses théories, notamment en poétique. La première se situe entre des conceptions naturelle et anthropologique du rythme. Pour définir une notion qui puisse aider à décrire les modes de signification dans les œuvres littéraires, il importe peu de savoir si, à l'origine, le sens de rhuthmoss'est développé depuis l'observation de la nature ou si l'application du terme aux phénomènes naturels est venue tardivement. II importe surtout de ne pas subordonner les rythmes discursifs aux périodicités naturelles ou physiologiques afin de pouvoir les considérer dans leur spécificité. Par contre, si on ne peut admettre que la production de rythmes dans les œuvres soit entièrement conditionnée par le "métronome» du cœur ou le "soufflet" de la poitrine, il ne faut pas non plus récuser la possibilité d'une relation entre l'appréhension (dans la poïesis ou dans la réception) de ces rythmes et celle du corps propre. La seconde opposition sépare les conceptions métaphysique et phénoménologique du rythme. La première fait appel à une loi antérieure à toute manifestation; la seconde conçoit le rythme comme coextensif au surgissement d'une forme. Le rhuthmos ionien, tel qu'explicité par Benveniste, oriente vers cette dernière voie et permet d'envisager, en poétique, l'étroite relation qui l'unit à la production du sens. Cette distinction permet de revenir à la première opposition: si l'on considère le rythme dans son apparaître, on peut dire que phénomènes naturels et œuvres humaines disposent des rythmes en tant qu'ils se manifestent. Toutefois, le rythme discursif, parce qu'il ne se produit que concurremment à une organisation signifiante, aura nécessairement une dimension anthropologique et historique.

Lucie Bourassa Université Laval 